\title{
Association between resting heart rate and cardiovascular risk factors in adolescents
}

\author{
Breno Quintella Farah ${ }^{1}$ • Diego Giulliano Destro Christofaro ${ }^{2}$ • P. Babu Balagopal ${ }^{3}$. \\ Bruno Remigio Cavalcante ${ }^{1}$ - Mauro Virgílio Gomes de Barros ${ }^{1}$. \\ Raphael Mendes Ritti-Dias ${ }^{1,4}$
}

Received: 2 April 2015 /Revised: 2 June 2015 / Accepted: 8 June 2015 / Published online: 24 June 2015

(C) Springer-Verlag Berlin Heidelberg 2015

\begin{abstract}
This cross-sectional study was performed in 2011 and included 4619 Brazilian adolescents (14-19 years old) to analyze the association between elevated resting heart rate (RHR) and cardiovascular risk factors in boys and girls. RHR and blood pressure were measured using an oscillometric monitor. Overweight was assessed by body mass index and abdominal obesity by waist circumference. Physical activity levels and sedentary behaviors were obtained using a questionnaire. The effect of clustering of cardiovascular risk factors on RHR was analyzed. For boys, abdominal obesity $(b=0.106, p=0.003)$, high sedentary behavior $(b=0.099, b<0.001)$, physical inactivity $(b=0.049, p=0.034)$, and high blood pressure $(b=0.160$, $p<0.001$ ) were associated with RHR, whereas for girls, only high blood pressure was associated with RHR ( $b=$ $0.259, p<0.001$ ), after adjustment for age, period of the day, and other cardiovascular risk factors. Boys with five risk factors presented significantly higher $(p<0.05)$ RHR values $\left(82.5 \pm 13.4\right.$ beats $\left.\min ^{-1}\right)$ than those for boys with-
\end{abstract}

Communicated by Peter de Winter

Raphael Mendes Ritti-Dias

raphaelritti@gmail.com

Breno Quintella Farah

brenofarah@hotmail.com

Diego Giulliano Destro Christofaro

ddcleite@yahoo.com.br

P. Babu Balagopal

BBalagop@nemours.org

Bruno Remigio Cavalcante

br_13remigio@hotmail.com

Mauro Virgílio Gomes de Barros

maurovgb@gmail.com out any cardiovascular risk factors (68.8 \pm 10.4 beats $\left.\min ^{-1}\right)$. The girls with five risk factors presented a mean RHR value of $89.8 \pm 9.9$ beats $\min ^{-1}$ that was higher $(p<0.05)$ than that for girls who had no risk factors $\left(79.6 \pm 10.9\right.$ beats $\left.\min ^{-1}\right)$.

Conclusions: Our study demonstrated that while RHR was associated with cardiovascular risk factors in both sexes, the clustering of risk factors amplified the elevation of RHR in a gender-dependent fashion.

\section{What is Known:}

- Resting heart rate is a marker of cardiovascular disease and mortality in adults and associated with risk factor such as higher levels of blood pressure, triglycerides, glucose, and obesity in children and adolescents.

What is New:

- The data from the current study suggest that the risk factor clustering is associated with elevated resting heart rate in adolescents and that the clustering of risk factors amplifies the elevation of resting heart rate in a gender-dependent fashion.
1 Graduate Program in Physical Education, University of Pernambuco, Recife, PE, Brazil

2 Laboratory of Investigation in Exercise - LIVE, Department of Physical Education, University of Paulista State (UNESP), Presidente Prudente, SP, Brazil

Nemours Children's Clinic and Mayo Clinic College of Medicine, Jacksonville, FL, USA

4 Albert Einstein Hospital, Albert Einstein Avenue, 627, 05652-900 Sao Paulo, SP, Brazil 
Keyword Heart rate - Risk factor · Cardiovascular disease . Adolescent health

\author{
Abbreviations \\ BMI Body mass index \\ CVD Cardiovascular disease \\ RHR Resting heart rate
}

\section{Introduction}

Resting heart rate (RHR) has been proposed as a simple measure that reflects the integrity of the autonomic nervous system. RHR is emerging as an easily measurable clinical parameter that is a predictive marker of cardiovascular disease (CVD) and mortality [19-21, 42]. Elevated RHR is associated with higher levels of blood pressure, abdominal obesity, triglycerides, and glucose in children and adolescents $[12,17,18,22$, 28 ], indicating its potential as a screening tool of cardiovascular risk in children.

Previous studies [12, 17, 18, 22, 28] have analyzed the association between cardiovascular risk factors and elevated RHR in adolescents, but several aspects remain less clear. For example, previous studies rarely considered the role of clustering of cardiovascular risk factors and its relationship with RHR in children and adolescents. In fact, clustering of risk factors for CVD is a reliable tool for assessing abnormal vascular function and may have more predictive power than when they are considered individually [3, 37]. The Pathobiological Determinants of Atherosclerosis in Youth risk score has been shown to predict the likelihood of advanced atherosclerotic lesions in the coronary arteries of youth (15-34 years old) [31]. Risk factor clustering has also been associated with abnormal vascular structure and function in youth [36]. It has also been reported that both the RHR [26, 28] and clustering of cardiovascular risk factors behave in a gender-dependent way [1]. It remains less clear if the association between the clustering of cardiovascular risk factors and RHR is also different between genders.

The aim of the current study was to analyze in a large sample of boys and girls the potential gender dichotomy in the association between elevated RHR and clustering of cardiovascular risk factors. We hypothesized that while individual cardiovascular risk factors are associated with elevated RHR, these associations will become stronger when they are clustered together and that the strength of this association differs between genders.

\section{Methods}

\section{Study design and sample}

This cross-sectional study was approved by the ethical committee of the University of Pernambuco in compliance with the Brazilian National Research Ethics System Guidelines. The target population consisted of highschool students between 14 and 19 years old from the public school system in the state of Pernambuco (northeast Brazil), which encompasses about $80 \%$ of all students attending this level of schooling (approximately 350,000 students).

The population sample in the current study was balanced in terms of geographical distribution, school size, and the period of the day that students attended school. Geographical distribution was determined by considering the number of students enrolled in each of the 17 school districts distributed throughout the Pernambuco State. School size was divided into three categories according to the number of students attending each high school as follows: small, <200; medium, 200 to 499; and large, $\geq 500$. The period of the day that students attended school was divided into two categories: daytime and evening. Taking these criteria into consideration, a two-stage cluster sampling procedure was performed to select the required sample. In the first stage, there was the stratification of schools by school district. In the second stage, there was the stratification by school size and period of the day. Selection was performed after generating random numbers using SPSS/PASW version 20 (IBM Corp., NY, USA), and class was used as the sampling unit for the final stage of the process.

\section{Data collection}

Data collection was performed between May and October in 2011. Demographic data, physical activity level, and sedentary behavior were obtained using an adapted version of the Global School-based Student Health Survey. This questionnaire has been widely used in epidemiological studies with adolescents. In previous studies in similar populations, we have reported concordance coefficient (kappa test) between 0.52 and 1.00 for a questionnaire [15, 38, 40]. In addition, evaluation of RHR, blood pressure, and obesity indicators was performed.

\section{Outcomes}

\section{Resting heart rate}

RHR was measured using the Omron HEM 742 blood pressure monitoring device (Omron, Shanghai, China) after the adolescents rested and remained seated for $5 \mathrm{~min}$. All measurements were performed three times in a seated position. 
The mean value of the last two measurements was used for analysis. Previous study has demonstrated good agreement between heart rate obtained using the Omron HEM 742 blood pressure monitoring device and a heart rate monitor [10]. RHR was categorized into normal (first to third quartile) and elevated (fourth quartile) RHR.

\section{Predictors}

\section{Physical activity level}

Physical activity level was assessed by the question "During a typical week on how many days were you physically active for a total of at least 60 min per day?" Adolescents were classified as active (if the answer was five or more days per week with at least $60 \mathrm{~min}$ per day of moderate to vigorous physical activity, i.e., $\geq 300 \mathrm{~min} / \mathrm{week}$ ) or insufficiently active ( $<300 \mathrm{~min} /$ week) [9]. Reproducibility indicators (i.e., testretest consistency, 1-week apart) showed the kappa coefficient to be 0.60 and Spearman's rank correlation coefficient to be 0.82 .

\section{Sedentary behaviors}

Sedentary behavior was assessed by the question "In a normal day, how much time you spend sitting, watching television, use computers, video games, chatting with friends, playing cards or dominoes?" Adolescents were classified as low sedentary behavior ( $<3 \mathrm{~h} /$ day) or high sedentary behavior $(\geq 3 \mathrm{~h} /$ day). Reproducibility indicators showed a kappa coefficient of 0.43 and Spearman's rank correlation coefficient of 0.53.

\section{Blood pressure}

Blood pressure was measured using the Omron HEM 742 blood pressure monitoring device (Omron, Shanghai, China) [10] after the adolescents rested and remained seated with legs uncrossed for 5 min. Appropriate cuff size was used for each adolescent. All blood pressure measurements were performed three times in the right arm placed at heart level in a seated position. The mean value of the last two measurements was used for analysis. High blood pressure was defined as systolic and/or diastolic blood pressure equal or higher than the reference sex, age, and height-specific 95th percentile [14].

\section{Overweight and obesity measures}

Adolescents were weighed without shoes and coats on an automatic scale, and the height was measured using a stadiometer. Overweight was determined by Cole et al. [11]. Waist circumference was measured in the standing position at the level of the umbilicus using a constant tension tape. Abdominal obesity was determined by waist circumference above the 80th percentile for their sex and age [39].

\section{Cluster of cardiovascular risk factors}

Clustering of cardiovascular risk factors was considered to be the sum of abdominal obesity, overweight, high sedentary behavior, insufficient level of physical activity, and high blood pressure. Only adolescents without missing data were considered for analysis. The cluster score ranged from 0 to 5 .

\section{Statistical analysis}

Data entry was conducted using the EpiData software package. Electronic data control was confirmed by means of the "Check" function. To detect errors, data entry was repeated and errors that were detected by the duplicate file comparison function were corrected. All statistical analyses were performed using SPSS/PASW version 20 (IBM Corp., NY, USA).

Continuous variables were summarized as mean and standard deviation, whereas categorical variables were summarized as relative frequency. Adolescents with normal and elevated RHR were compared using independent $t$ test for continuous variables and chi-square test for categorical variables.

Linear regression modeling was used to analyze the association between elevated RHR and cardiovascular risk factors stratified by sex. At first, the crude analyzes were performed and subsequently adjusted for age, period of the day, and all cardiovascular risk factors. A one-way analysis of variance, followed by Newman-Keuls post hoc test, was used to compare RHR values with the number of cardiovascular risk factors ( 0 to 5 ). A $p$ value $<0.05$ was considered statistically significant for all the analyses.

\section{Results}

Eighty-five schools were visited in 48 cities in the state of Pernambuco (in the northeast region of Brazil). In the selected schools, a total of 7195 students were evaluated, and among those, 919 were out of the target age group (20 years or older), and 12 questionnaires were excluded because of inconsistent data and incompleteness. Thus, a total of 6264 were included. However, 1645 adolescents missed RHR data. Thus, the final sample in the present study consisted of 4619 adolescents with 1830 boys and 2789 girls. Characteristics of adolescents are shown in Table 1. Girls showed higher RHR than boys $(p<0.05)$.

Among the adolescents, $21.8 \%$ had abdominal obesity, $15.7 \%$ were overweight, $20 \%$ had high sedentary behavior, 
Table 1 General characteristics of adolescents according to sexes in Brazil in $2011(n=4619)$

\begin{tabular}{|c|c|c|c|c|}
\hline & Total & Boys $(n=1830)$ & Girls $(n=2789)$ & $p$ value \\
\hline Age (years) & $16.6 \pm 1.3$ & $16.7 \pm 1.3$ & $16.5 \pm 1.3$ & $<0.001$ \\
\hline \multicolumn{5}{|l|}{ Ethnicity (\%) } \\
\hline White & 27.5 & 28.1 & 27.1 & $<0.001$ \\
\hline Black & 10.6 & 13.8 & 8.5 & $<0.001$ \\
\hline Brown & 50.8 & 47.7 & 53.0 & $<0.001$ \\
\hline Other & 10.9 & 10.2 & 11.3 & $<0.001$ \\
\hline Resting heart rate (beats $\min ^{-1}$ ) & $77.6 \pm 12.7$ & $72.4 \pm 11.8$ & $81.1 \pm 12.0$ & $<0.001$ \\
\hline Abdominal obesity (\%) & 21.8 & 14.6 & 26.4 & $<0.001$ \\
\hline Overweight $(\%)$ & 15.7 & 15.2 & 15.9 & 0.508 \\
\hline High sedentary behavior ( $\%)$ & 50.9 & 38.4 & 61.6 & 0.085 \\
\hline Insufficiently active (\%) & 66.8 & 35.9 & 64.1 & $<0.001$ \\
\hline High blood pressure (\%) & 16.3 & 61.3 & 38.7 & $<0.001$ \\
\hline \multicolumn{5}{|l|}{ Cluster of CV risk factors $(\%)$} \\
\hline None & 10.9 & 12.9 & 9.5 & $<0.001$ \\
\hline One & 35.6 & 36.7 & 34.8 & $<0.001$ \\
\hline Two & 33.4 & 32.2 & 34.1 & $<0.001$ \\
\hline Three & 12.5 & 10.8 & 13.6 & $<0.001$ \\
\hline Four & 5.5 & 4.7 & 6.0 & $<0.001$ \\
\hline Five & 1.8 & 2.3 & 1.4 & $<0.001$ \\
\hline
\end{tabular}

Values expressed as mean (standard deviation) and frequency

$66.8 \%$ were insufficiently active, and $16.3 \%$ had high blood pressure. In boys, abdominal obesity, overweight, high sedentary behavior, insufficient activity level, and high blood pressure were positively associated with RHR $(p<0.05)$. However, after statistical adjustments for age, period of the day, and all cardiovascular risk factors, abdominal obesity, high sedentary behavior, physical inactivity, and high blood pressure remained associated with RHR $(p<0.05)$. For girls, only high blood pressure remained significantly associated with RHR and the association remained significant after the adjustments (Table 2).

Nearly $13 \%$ of boys had no cardiovascular risk factors, while $36.8,32.3,10.8,4.7$, and $2.4 \%$ showed $1,2,3,4$, and 5 cardiovascular risk factors, respectively. About $9.5 \%$ of girls had no cardiovascular risk factors, while 35.0, 34.3, 13.6, 6.0, and $1.0 \%$ showed 1, 2, 3, 4, and 5 cardiovascular risk factors, respectively. There was a significant association between the clustering of cardiovascular risk factors with RHR in both sexes $(p<0.001)$. Figure 1 shows the mean and standard deviation of RHR values stratified by the number of cardiovascular risk factors in the cluster in boys and girls. In boys, those with five risk factors presented RHR values of $82.5 \pm 13.4$ beats $\mathrm{min}^{-1}$, which was significantly higher $(p<0.05)$ than that for boys without any cardiovascular risk factors $\left(68.8 \pm 10.4\right.$ beats $\left.\mathrm{min}^{-1}\right)$. The girls with five risk factors presented a mean RHR value of $89.8 \pm 9.9$ beats $\mathrm{min}^{-1}$ that was higher $(p<0.05)$ than girls who had no risk factors $(79.6 \pm$ 10.9 beats $\left.\mathrm{min}^{-1}\right)$.

\section{Discussion}

A notable finding of this large population-based study in adolescents is the amplification of RHR with an increasing number of risk factors for CVD in a cluster of risk factors. Interestingly, the elevation in RHR appears to be gender dependent. In boys, abdominal obesity, high sedentary behavior, physical inactivity, and high blood pressure were independently associated with elevated RHR. However, there was a linear increase in RHR in boys with the increase in the number of risk factors clustered together. In girls, only high blood pressure was independently associated with elevated RHR, but a cluster consisting of three or more cardiovascular risk factors was associated with a higher RHR compared to girls without any risk factors of CVD.

In boys, abdominal obesity and overweight were associated positively with RHR, but only abdominal obesity remained significant after adjustments for age, period of the day, and other cardiovascular risk factors. Kwok et al. [28] also found a positive association between RHR and waist circumference in boys. While a previous study observed an association between waist circumference and 
Table 2 Crude and adjusted analyses of the relationship between resting heart rate and cardiovascular risk factors in adolescents

\begin{tabular}{|c|c|c|c|c|}
\hline Independent variables & Models & $\beta^{\mathrm{a}}(\mathrm{SE})$ & $b^{\mathrm{b}}$ & $p$ \\
\hline \multicolumn{5}{|l|}{ Boys } \\
\hline \multirow[t]{2}{*}{ Abdominal obesity $(\mathrm{no}=0$, yes $=1)$} & Crude & $4.42(0.78)$ & 0.132 & $<0.001$ \\
\hline & Adjusted & $3.55(1.21)$ & 0.106 & 0.003 \\
\hline \multirow[t]{2}{*}{ Overweight $($ no $=0$, yes $=1)$} & Crude & $3.25(0.77)$ & 0.099 & $<0.001$ \\
\hline & Adjusted & $-0.52(1.19)$ & -0.016 & 0.662 \\
\hline \multirow[t]{2}{*}{ High sedentary behavior $($ no $=0$, yes $=1)$} & Crude & $2.87(0.55)$ & 0.121 & $<0.001$ \\
\hline & Adjusted & $2.33(0.55)$ & 0.099 & $<0.001$ \\
\hline \multirow[t]{2}{*}{ Insufficiently active $($ no $=0$, yes $=1)$} & Crude & $1.44(0.57)$ & 0.059 & 0.011 \\
\hline & Adjusted & $1.17(0.55)$ & 0.049 & 0.034 \\
\hline \multirow[t]{2}{*}{ High blood pressure $($ no $=0$, yes $=1$ ) } & Crude & $4.63(0.63)$ & 0.169 & $<0.001$ \\
\hline & Adjusted & $4.37(0.64)$ & 0.160 & $<0.001$ \\
\hline \multicolumn{5}{|l|}{ Girls } \\
\hline \multirow[t]{2}{*}{ Abdominal obesity $(\mathrm{no}=0, \mathrm{yes}=1)$} & Crude & $0.67(0.51)$ & 0.025 & 0.197 \\
\hline & Adjusted & $0.05(0.64)$ & 0.002 & 0.934 \\
\hline \multirow[t]{2}{*}{ Overweight $(\mathrm{no}=0$, yes $=1)$} & Crude & $1.10(0.62)$ & 0.034 & 0.076 \\
\hline & Adjusted & $-0.37(0.78)$ & -0.011 & 0.636 \\
\hline \multirow[t]{2}{*}{ High sedentary behavior $($ no $=0$, yes $=1)$} & Crude & $-0.21(0.45)$ & -0.009 & 0.642 \\
\hline & Adjusted & $-0.52(0.44)$ & -0.022 & 0.241 \\
\hline \multirow[t]{2}{*}{ Insufficiently active (no=0, yes $=1)$} & Crude & $0.81(0.50)$ & 0.031 & 0.103 \\
\hline & Adjusted & $0.60(0.48)$ & 0.023 & 0.213 \\
\hline \multirow[t]{2}{*}{ High blood pressure $($ no $=0$, yes $=1)$} & Crude & $10.13(0.72)$ & 0.259 & $<0.001$ \\
\hline & Adjusted & $10.08(0.73)$ & 0.258 & $<0.001$ \\
\hline
\end{tabular}

Adjusted for age, period of the day, and all cardiovascular risk factors

$S E$ standard error

${ }^{a}$ Regression coefficient

${ }^{\mathrm{b}}$ Standardized beta coefficients lower parasympathetic modulation to the heart, there was no such association for body mass index (BMI) [16, 29], which is in agreement with the results of the current study. Since waist circumference represents visceral adiposity more closely than BMI [5], these results suggest that the abdominal obesity has a stronger negative impact on RHR. Although the mechanisms of these responses have not been evaluated in the current study, it is known that fat cells are responsible for secreting various adipokines, and among them is leptin, which is responsible for activating the neural pathways that increase the activity of the sympathetic nervous system $[6,13]$.

The current study also examined the association between sedentary behavior and RHR in adolescents. In boys, high sedentary behavior showed a significant association with higher RHR, independent of age, abdominal obesity, low physical activity level, overweight, and high blood pressure. Studies in adults have shown that structural changes in the left
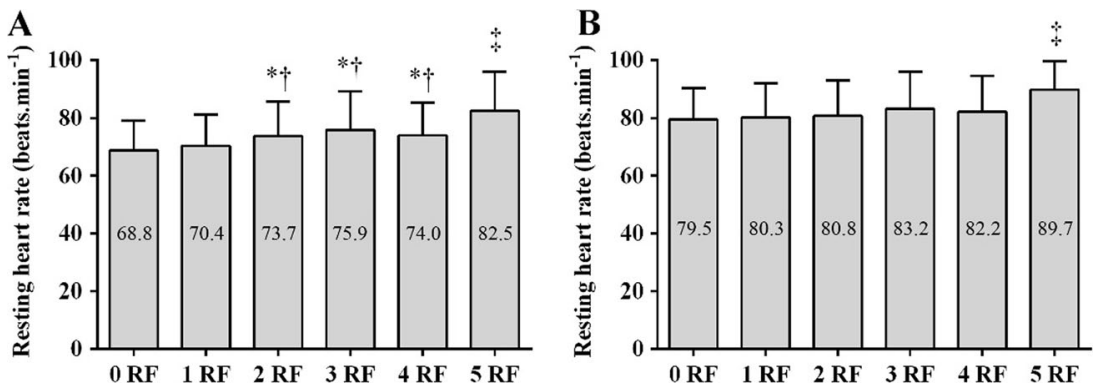

Fig. 1 Mean and standard deviation of resting heart rate values stratified by the number of risk factors included in the cluster in boys (a) and girls (b), in Brazil in 2011. $R F$ risk factor. ${ }^{*} p<0.05$, statistically different from zero RF and one RF; $₫ p<0.05$, statistically different from all the clusters 
ventricle are associated with television viewing time [23]. Another possible contributing factor is the lifestyle (nutritional and physical activity habits) of the adolescents. In fact, adolescents with sedentary lifestyles are more likely to consume more soft drinks, sweets, cookies, and processed meat that have high amounts of sodium, glucose, and caffeine [8]. In addition, adolescents with high sedentary behavior present a worse lipid profile [41] that may be associated with a shift in cardiac autonomic modulation toward higher sympathetic and lower parasympathetic modulation to the heart [30]. However, future more directed studies to confirm these ideas and the underlying mechanisms are needed.

Approximately $70 \%$ of adolescents in the current study presented two or more cardiovascular risk factors, and the clustering of cardiovascular risk factors increased the likelihood of adolescents having an elevated RHR. Various studies have suggested a close relationship between the clustering of cardiovascular risk factors and higher levels of inflammatory markers, insulin resistance, worse lipid profile, and lower cardiorespiratory fitness $[2,7]$. These factors have also been associated with increased sympathetic modulation to the heart, likely leading to elevated RHR [24, 25]. Interestingly, for boys, there is a linear increase in RHR with the increasing number of cardiovascular risk factors, while for girls, only the clusters of three or more cardiovascular risk factors were associated with higher RHR. Previous studies in adults have also shown such gender differences, but they diminished after middle age, perhaps attributable to the influence of female sex hormones on the autonomic activity [27, 32, 34]. However, such studies in children are scarce.

Clustering of risk factors of CVD has been suggested as a useful tool for assessing abnormal vascular function in adults and youth [4, 36]. In this context, the amplification of RHR with increasing number of risk factors for CVD in the cluster found in our study has clinical significance. RHR is an easily measurable clinical parameter. Although the aim of this study was not to define an optimal RHR for a given individual, in the current study, we have observed that boys with higher number of risk factors had a mean value of 83 beats $\mathrm{min}^{-1}$, while girls with higher risk presented a mean value of 90 beats $\mathrm{min}^{-1}$. These results suggest that the adolescents with values in the ranges noted above may require early attention to their cardiovascular health. Prospective longitudinal studies are warranted to shed more light on these cutoff values and the potential implications of the data from the current study on the notion that girls live longer and develop CVD usually at a later age than boys.

The relatively large sample size and the fairly narrow age range are the strength of this study. In addition, strict sampling procedures were established to ensure that the sample was representative of the adolescents in state of Brazil. There are also certain limitations of this study that need to be pointed out. The cross-sectional design precludes proof of causality that enhanced RHR directly leads to changes in the vasculature early in adolescents. Longitudinal evaluations in multiethnic adolescent populations are needed to make these conclusions. The use of self-reported measures for physical activity and sedentary behavior and the lack of objective vascular measures are important limitations that need to be considered. The reproducibility of the reliability coefficients for the objectively measured sedentary behaviors in the current study was only moderately reproducible but was similar to previous studies in adolescents $[33,35]$. Unfortunately, we could not objectively assess the pubertal status of the participants in this study. However, the participants had a narrow age range, which could help minimize the potential effects of pubertal differences on the measured parameters. Other risk factors for CVD such as cardiorespiratory fitness, insulin resistance, and inflammatory markers could not be included in this population-based study.

In conclusion, our results show that an increase in the number of cardiovascular risk factors in a cluster of risk factors amplifies RHR in a gender-dependent manner. Despite its simplicity, RHR has been a seriously neglected clinical tool in the assessment of cardiovascular risk in children and adolescents. The current study suggests the promise of this simple tool as an integral part of clinical care for adolescents and to identify those who are at risk for early CVD and/or atherosclerosis.

Acknowledgments This work was supported by a grant (grant \#481067/2010-8) from the Brazilian National Council for Scientific and Technological Development (CNPq). Additional support was provided by the Brazilian Federal Agency for Support and Evaluation of Graduate Education (CAPES).

BQ Farah was responsible for starting the writing of the manuscript. In addition, no person received any honorarium, grant, or other forms of payment to produce the manuscript.

Breno Quintella Farah conceptualized and designed the study, collected data, carried out the analyses, and drafted the initial manuscript.

Diego Giulliano Destro Christofaro conceptualized and designed the study, carried out the analyses, and reviewed the manuscript.

P. Babu Balagopal contributed to the interpretation of the data and reviewed the manuscript.

Bruno Remígio Cavalcante collected data and reviewed the manuscript.

Mauro Virgílio Gomes Barros designed the data collection instruments, coordinated and supervised data collection, and reviewed the manuscript.

Raphael M. Ritti-Dias designed the data collection instruments, coordinated and supervised data collection, conceptualized and designed the study, and reviewed the manuscript.

All the authors approved the final manuscript as submitted and agreed to be accountable for all aspects of the work.

Conflict of interest The authors declare that they have no competing interests. 


\section{References}

1. Alamian A, Paradis G (2009) Clustering of chronic disease behavioral risk factors in Canadian children and adolescents. Prev Med 48:493-499

2. Andersen LB, Muller K, Eiberg S, Froberg K, Andersen JF, Bugge A, Hermansen Bel N, McMurray RG (2010) Cytokines and clustered cardiovascular risk factors in children. Metabolism 59:561566

3. Balagopal PB, de Ferranti SD, Cook S, Daniels SR, Gidding SS, Hayman LL, McCrindle BW, Mietus-Snyder ML, Steinberger J (2011) Nontraditional risk factors and biomarkers for cardiovascular disease: mechanistic, research, and clinical considerations for youth: a scientific statement from the American Heart Association. Circulation 123:2749-2769

4. Berenson GS, Srinivasan SR, Bao W, Newman WP, Tracy RE, Wattigney WA (1998) Association between multiple cardiovascular risk factors and atherosclerosis in children and young adults. The Bogalusa Heart Study. N Engl J Med 338:1650-1656

5. Brambilla P, Bedogni G, Moreno LA, Goran MI, Gutin B, Fox KR, Peters DM, Barbeau P, De Simone M, Pietrobelli A (2006) Crossvalidation of anthropometry against magnetic resonance imaging for the assessment of visceral and subcutaneous adipose tissue in children. Int $\mathrm{J}$ Obes (Lond) 30:23-30

6. Brydon L, O'Donnell K, Wright CE, Wawrzyniak AJ, Wardle J, Steptoe A (2008) Circulating leptin and stress-induced cardiovascular activity in humans. Obesity 16:2642-2647

7. Bugge A, El-Naaman B, McMurray RG, Froberg K, Nielsen CH, Muller K, Andersen LB (2012) Inflammatory markers and clustered cardiovascular disease risk factors in Danish adolescents. Horm Res Paediatr 78:288-296

8. Camelo LV, Rodrigues JF, Giatti L, Barreto SM (2012) Sedentary leisure time and food consumption among Brazilian adolescents: the Brazilian National School-Based Adolescent Health Survey (PeNSE), 2009. Cad Saude Publica 28:2155-2162

9. Cavill N, Bidlle S, Sallis J (2001) Health enhancing physical activity for young people: statement of United Kingdom expert consensus conference. Pediatr Exerc Sci 13:12-25

10. Christofaro DG, Casonatto J, Polito MD, Cardoso JR, Fernandes R, Guariglia DA, Gerage AM, de Oliveira AR (2009) Evaluation of the Omron MX3 Plus monitor for blood pressure measurement in adolescents. Eur J Pediatr 168:1349-1354

11. Cole TJ, Bellizzi MC, Flegal KM, Dietz WH (2000) Establishing a standard definition for child overweight and obesity worldwide: international survey. BMJ 320:1240-1243

12. Dong B, Wang Z, Wang HJ, Ma J (2015) The association between resting heart rate and blood pressure among children and adolescents with different waist circumferences. Eur J Pediatr 174:191-7

13. Eikelis N, Schlaich M, Aggarwal A, Kaye D, Esler M (2003) Interactions between leptin and the human sympathetic nervous system. Hypertension 41:1072-1079

14. Falkner B, Daniels SR (2004) Summary of the fourth report on the diagnosis, evaluation, and treatment of high blood pressure in children and adolescents. Hypertension 44:387-388

15. Farah BQ, Barros MV, Balagopal B, Ritti-Dias RM (2014) Heart rate variability and cardiovascular risk factors in adolescent boys. $\mathrm{J}$ Pediatr 165:945-950

16. Farah BQ, Prado WL, Tenorio TR, Ritti-Dias RM (2013) Heart rate variability and its relationship with central and general obesity in obese normotensive adolescents. Einstein (Sao Paulo) 11:285-290

17. Fernandes RA, Freitas Junior IF, Codogno JS, Christofaro DG, Monteiro HL, Roberto Lopes DM (2011) Resting heart rate is associated with blood pressure in male children and adolescents. $\mathrm{J}$ Pediatr 158:634-637
18. Fernandes RA, Vaz Ronque ER, Venturini D, Barbosa DS, Silva DP, Cogo CT, Carnelossi MS, Batista MB, Coelho E, Silva MJ, Sardinha LB, Cyrino ES (2013) Resting heart rate: its correlations and potential for screening metabolic dysfunctions in adolescents. BMC Pediatr 13:48

19. Fox K, Borer JS, Camm AJ, Danchin N, Ferrari R, Lopez Sendon JL, Steg PG, Tardif JC, Tavazzi L, Tendera M (2007) Resting heart rate in cardiovascular disease. J Am Coll Cardiol 50:823-830

20. Fox K, Bousser MG, Amarenco P, Chamorro A, Fisher M, Ford I, Hennerici MG, Mattle HP, Rothwell PM (2013) Heart rate is a prognostic risk factor for myocardial infarction: a post hoc analysis in the PERFORM (Prevention of cerebrovascular and cardiovascular Events of ischemic origin with teRutroban in patients with a history oF ischemic strOke or tRansient ischeMic attack) study population. Int J Cardiol 168:3500-3505

21. Fox KM, Ferrari R (2011) Heart rate: a forgotten link in coronary artery disease? Nat Rev Cardiol 8:369-379

22. Freitas Junior IF, Monteiro PA, Silveira LS, Cayres SU, Antunes BM, Bastos KN, Codogno JS, Sabino JP, Fernandes RA (2012) Resting heart rate as a predictor of metabolic dysfunctions in obese children and adolescents. BMC Pediatr 12:5

23. Gibbs BB, Reis JP, Schelbert EB, Craft LL, Sidney S, Lima J, Lewis CE (2014) Sedentary screen time and left ventricular structure and function: the CARDIA study. Med Sci Sports Exerc 46: 276-283

24. Gutin B, Barbeau P, Litaker MS, Ferguson M, Owens S (2000) Heart rate variability in obese children: relations to total body and visceral adiposity, and changes with physical training and detraining. Obes Res 8:12-19

25. Gutin B, Howe C, Johnson MH, Humphries MC, Snieder H, Barbeau P (2005) Heart rate variability in adolescents: relations to physical activity, fitness, and adiposity. Med Sci Sports Exerc 37: 1856-1863

26. Hallal PC, Menezes AM, Bertoldi AD, Dumith SC, Araujo CL (2010) Resting pulse rate among adolescents: the 11-year followup of the 1993 Pelotas (Brazil) birth cohort study. Cad Saude Publica 26:1963-1971

27. Krishna P, Rao D, Navekar VV (2013) Cardiac autonomic activity in overweight and underweight young adults. Indian J Physiol Pharmacol 57:146-152

28. Kwok SY, So HK, Choi KC, Lo AF, Li AM, Sung RY, Nelson EA (2013) Resting heart rate in children and adolescents: association with blood pressure, exercise and obesity. Arch Dis Child 98:287291

29. Lucini D, de Giacomi G, Tosi F, Malacarne M, Respizzi S, Pagani M (2013) Altered cardiovascular autonomic regulation in overweight children engaged in regular physical activity. Heart 99: 376-381

30. Mancia G, Bousquet P, Elghozi JL, Esler M, Grassi G, Julius S, Reid J, Zwieten V, Peter A (2007) The sympathetic nervous system and the metabolic syndrome. J Hypertens 25:909-920, 910.1097/ HJH.1090b1013e328048d328004

31. McGill HC Jr, McMahan CA, Gidding SS (2008) Preventing heart disease in the 21st century: implications of the Pathobiological Determinants of Atherosclerosis in Youth (PDAY) study. Circulation 117:1216-1227

32. Ramaekers D, Ector H, Aubert AE, Rubens A, Van de Werf $F$ (1998) Heart rate variability and heart rate in healthy volunteers. Is the female autonomic nervous system cardioprotective? Eur Heart J 19:1334-1341

33. Raudsepp L, Neissaar I, Kull M (2008) Longitudinal stability of sedentary behaviors and physical activity during early adolescence. Pediatr Exerc Sci 20:251-262

34. Ryan SM, Goldberger AL, Pincus SM, Mietus J, Lipsitz LA (1994) Gender- and age-related differences in heart rate dynamics: are women more complex than men? J Am Coll Cardiol 24:1700-1707 
35. Schmitz Kathryn H, Harnack L, Fulton Janet E, Jacobs David R, Gao S, Lytle Leslie A, Van Coevering P (2004) Reliability and validity of a brief questionnaire to assess television viewing and computer use by middle school children. J Sch Health 74:370-377

36. Shah AS, Dolan LM, Gao Z, Kimball TR, Urbina EM (2011) Clustering of risk factors: a simple method of detecting cardiovascular disease in youth. Pediatrics 127:e312-318

37. Steinberger J, Daniels SR, Eckel RH, Hayman L, Lustig RH, McCrindle B, Mietus-Snyder ML (2009) Progress and challenges in metabolic syndrome in children and adolescents: a scientific statement from the American Heart Association Atherosclerosis, Hypertension, and Obesity in the Young Committee of the Council on Cardiovascular Disease in the Young; Council on Cardiovascular Nursing; and Council on Nutrition, Physical Activity, and Metabolism. Circulation 119:628-647

38. Tassitano RM, Barros MV, Tenorio MC, Bezerra J, Florindo AA, Reis RS (2010) Enrollment in physical education is associated with health-related behavior among high school students. J Sch Health $80: 126-133$

39. Taylor RW, Jones IE, Williams SM, Goulding A (2000) Evaluation of waist circumference, waist-to-hip ratio, and the conicity index as screening tools for high trunk fat mass, as measured by dual-energy X-ray absorptiometry, in children aged 3-19 y. Am J Clin Nutr 72: 490-495

40. Tenório Maria Cecília Marinho, Barros Mauro Virgilio Gomes de, Tassitano Rafael Miranda, Bezerra Jorge, Tenório Juliana Marinho, \& Hallal Pedro Curi (2010) Atividade física e comportamento sedentário em adolescentes estudantes do ensino médio. Revista Brasileira de Epidemiologia 13:105-117

41. Vaisto J, Eloranta AM, Viitasalo A, Tompuri T, Lintu N, Karjalainen P, Lampinen EK, Agren J, Laaksonen DE, Lakka HM, Lindi V, Lakka TA (2014) Physical activity and sedentary behaviour in relation to cardiometabolic risk in children: crosssectional findings from the Physical Activity and Nutrition in Children (PANIC) Study. Int J Behav Nutr Phys Act 11:55

42. Woodward M, Webster R, Murakami Y, Barzi F, Lam TH, Fang X, Suh I, Batty GD, Huxley R, Rodgers A (2012) The association between resting heart rate, cardiovascular disease and mortality : evidence from 112,680 men and women in 12 cohorts. Eur J Prev Cardiol 21:719-26 